\title{
PRODUCTS OF DERIVATIONS WHICH ACT AS LIE DERIVATIONS ON COMMUTATORS OF RIGHT IDEALS
}

\author{
V. DE FILIPPIS
}

Received 5 April 2006; Revised 22 October 2006; Accepted 30 October 2006

Let $R$ be a prime ring of characteristic different from $2, I$ a nonzero right ideal of $R, d$ and $\delta$ nonzero derivations of $R$, and $s_{4}\left(x_{1}, x_{2}, x_{3}, x_{4}\right)$ the standard identity of degree 4 . If the composition $(d \delta)$ is a Lie derivation of $[I, I]$ into $R$, then either $s_{4}(I, I, I, I) I=0$ or $\delta(I) I=d(I) I=0$.

Copyright (c) 2006 Hindawi Publishing Corporation. All rights reserved.

Throughout this note, $R$ will be always a prime ring of characteristic different from 2 with center $Z(R)$, extended centroid $C$, and two-sided Martindale quotient ring $Q$. Let $f: R \rightarrow R$ be additive mapping of $R$ into itself. It is said to be a derivation of $R$ if $f(x y)=$ $f(x) y+x f(y)$, for all $x, y \in R$. Let $S \subseteq R$ be any subset of $R$. If for any $x, y \in S, f([x, y])=$ $[f(x), y]+[x, f(y)]$, then the mapping $f$ is called a Lie derivation on $S$. Obviously any derivation of $R$ is a Lie derivation on any arbitrary subset $S$ of $R$.

A typical example of a Lie derivation is an additive mapping which is the sum of a derivation and a central map sending commutators to zero.

The well-known Posner first theorem states that if $\delta$ and $d$ are two nonzero derivations of $R$, then the composition $(d \delta)$ cannot be a nonzero derivation of $R$ [12, Theorem 1$]$. An analog of Posner's result for Lie derivations was proved by Lanski [8]. More precisely, Lanski showed that if $\delta$ and $d$ are two nonzero derivations of $R$ and $L$ is a Lie ideal of $R$, then $(d \delta)$ cannot be a Lie derivation of $L$ into $R$ unless $\operatorname{char}(R)=2$ and either $R$ satisfies $s_{4}\left(x_{1}, \ldots, x_{4}\right)$, the standard identity of degree 4 , or $d=\alpha \delta$, for $\alpha \in C$.

This note is motived by the previous cited results. Our main theorem gives a generalization of Lanski's result to the case when $(d \delta)$ is a Lie derivation of the subset $[I, I]$ into $R$, where $I$ is a nonzero right ideal of $R$ and the characteristic of $R$ is different from 2 . The statement of our result is the following.

THEOREM 1. Let $R$ be a prime ring of characteristic different from 2, I a nonzero right ideal of $R, d$ and $\delta$ nonzero derivations of $R$, and $s_{4}\left(x_{1}, \ldots, x_{4}\right)$ the standard identity of degree 4 . If the composition $(d \delta)$ is a Lie derivation of $[I, I]$ into $R$, then either $s_{4}(I, I, I, I) I=0$ or $\delta(I) I=d(I) I=0$. 
Remark 2. Notice that for all $u, v \in[I, I]$, we obviously have that

$$
(d \delta)([u, v])=[(d \delta)(u), v]+[u,(d \delta)(v)]+[\delta(u), d(v)]+[d(u), \delta(v)]
$$

Hence, since we suppose that $(d \delta)$ is a Lie derivation on $[I, I]$, we will always assume as a main hypothesis that $[\delta(u), d(v)]+[d(u), \delta(v)]=0$, for any $u, v \in[I, I]$.

Remark 3. The assumption $S_{4}(I, I, I, I) I \neq 0$ is essential to the main result. For example, consider $R=M_{3}(F)$, for $F$ a field of characteristic 3, and let $e_{i j}$ be the usual matrix unit in $R$. Let $I=\left(e_{11}+e_{22}\right) R, \delta$ the inner derivation induced by the element $e_{13}, d$ the inner derivation induced by the element $e_{12}$, that is, $\delta(x)=\left[e_{13}, x\right]=e_{13} x-x e_{13}$, and $d(x)=$ $\left[e_{12}, x\right]=e_{12} x-x e_{12}$, for all $x \in R$. In this case, notice that $S_{4}\left(x_{1}, x_{2}, x_{3}, x_{4}\right) x_{5}$ is an identity for $I$, moreover

$$
\begin{aligned}
{\left[\delta\left(\left[\left(e_{11}+e_{22}\right) x_{1},\left(e_{11}+e_{22}\right) x_{2}\right]\right), d\left(\left[\left(e_{11}+e_{22}\right) y_{1},\left(e_{11}+e_{22}\right) y_{2}\right]\right)\right] } \\
\quad+\left[d\left(\left[\left(e_{11}+e_{22}\right) x_{1},\left(e_{11}+e_{22}\right) x_{2}\right]\right), \delta\left(\left[\left(e_{11}+e_{22}\right) y_{1},\left(e_{11}+e_{22}\right) y_{2}\right]\right)\right] \\
=\left(d\left(\left[\left(e_{11}+e_{22}\right) x_{1},\left(e_{11}+e_{22}\right) x_{2}\right]\right)\left[\left(e_{11}+e_{22}\right) y_{1},\left(e_{11}+e_{22}\right) y_{2}\right]\right) e_{13} \\
\quad-\left(d\left(\left[\left(e_{11}+e_{22}\right) y_{1},\left(e_{11}+e_{22}\right) y_{2}\right]\right)\left[\left(e_{11}+e_{22}\right) x_{1},\left(e_{11}+e_{22}\right) x_{2}\right]\right) e_{13}=0
\end{aligned}
$$

for any $x_{1}, x_{2}, y_{1}, y_{2} \in R$, but clearly $d(I) I=\left[e_{12}, I\right] I \neq 0$.

In the particular case $I=R$ and both $d, \delta$ are inner derivations, induced, respectively, by some elements $a, b \in R$, our theorem has the following flavor.

Lemмa 4. Let $R$ be a prime ring of characteristic different from $2, a, b \in R$ such that $[[a, v]$, $[b, u]]+[[b, v],[a, u]]=0$, for all $v, u \in[R, R]$. Then either $a$ is a central element of $R$ or $b$ is a central one.

The proof is a clear special case of [8, Theorem 6].

We first fix some notations and recall some useful facts.

Remark 5. Denote by $T=Q *_{C} C\{X\}$ the free product over $C$ of the $C$-algebra $Q$ and the free $C$-algebra $C\{X\}$, with $X$ a countable set consisting of noncommuting indeterminates $\left\{x_{1}, \ldots, x_{n}\right\}$. The elements of $T$ are called generalized polynomials with coefficients in $Q$. $I, I R$, and $I Q$ satisfy the same generalized polynomial identities with coefficients in $Q$. For more details about these objects, we refer the reader to $[1,2,4]$.

Remark 6. Any derivation of $R$ can be uniquely extended to a derivation of $Q$, and so any derivation of $R$ can be defined on the whole of $Q$ [2, Proposition 2.5.1]. Moreover $Q$ is a prime ring as well as $R$ and the extended centroid $C$ of $R$ coincides with the center of $Q$ [2, Proposition 2.1.7, Remark 2.3.1].

Remark 7. Let $f\left(x_{1}, \ldots, x_{n}, d\left(x_{1}\right), \ldots, d\left(x_{n}\right)\right)$ be a differential identity of $R$. One of the following holds (see [7]):

(1) either $d$ is an inner derivation in $Q$, in the sense that there exists $q \in Q$ such that $d(x)=[q, x]$, for all $x \in Q$ and $Q$ satisfies the generalized polynomial identity $f\left(x_{1}, \ldots, x_{n},\left[q, x_{1}\right], \ldots,\left[q, x_{n}\right]\right)$; 
(2) or $R$ satisfies the generalized polynomial identity

$$
f\left(x_{1}, \ldots, x_{n}, y_{1}, \ldots, y_{n}\right) \text {. }
$$

Moreover $I, I R$, and IQ satisfy the same differential identities with coefficients in $Q$ (see [9]).

Finally, as a consequence of [11, Theorem 2], we have the following.

Remark 8. Let $R$ be a prime ring and $\sum_{i=1}^{m} a_{i} X b_{i}+\sum_{j=1}^{n} c_{j} X d_{j}=0$, for all $X \in R$, where $a_{i}, b_{i}, c_{j}, d_{j} \in R C$. If $\left\{a_{1}, \ldots, a_{m}\right\}$ are linearly $C$-independent, then each $b_{i}$ is $C$-dependent on $d_{1}, \ldots, d_{n}$. Analogously, if $\left\{b_{1}, \ldots, b_{m}\right\}$ are linearly $C$-independent, then each $a_{i}$ is $C$ dependent on $c_{1}, \ldots, c_{n}$.

For the remainder of the note we will assume that the hypothesis of the theorem holds but that the conclusion is false.

Thus, we will always suppose that there exist $c_{1}, c_{2}, c_{3}, c_{4}, c_{5}, c_{6}, c_{7}, c_{8}, c_{9} \in I$ such that $s_{4}\left(c_{1}, c_{2}, c_{3}, c_{4}\right) c_{5} \neq 0$, and either $\delta\left(c_{6}\right) c_{7} \neq 0$ or $d\left(c_{8}\right) c_{9} \neq 0$.

We begin with the following.

Lemma 9. Let $\delta$ and $d$ both be Q-inner derivations such that either $\delta(I) I \neq 0$ or $d(I) I \neq 0$. Then $R$ is a ring satisfying a nontrivial generalized polynomial identity.

Proof. By Remark 2, we assume that $[\delta(u), d(v)]+[d(u), \delta(v)]=0$, for any $u, v \in[I, I]$. Let $a, b \in Q$ such that $\delta(x)=[a, x]$ and $d(x)=[b, x]$, for all $x \in R$.

Without loss of generality, we may assume in this context that $\delta(I) I \neq 0$. Notice that if $\{y, a y\}$ are linearly $C$-dependent for all $y \in I$, then there exists $\alpha \in C$, such that $(a-\alpha) I=0$ (see [10, Lemma 3]). If we replace $a$ by $a-\alpha$, since they induce the same inner derivation, it follows that $\delta(I) I=[a-\alpha, I] I=0$, a contradiction. Thus there exists $x \in I$ such that $\{x, a x\}$ are linearly $C$-independent.

Let $x \in I$ such that $\{x, a x\}$ are linearly $C$-independent and $r_{1}, r_{2}, r_{3}, r_{4}$ are any elements of $R$. Then

$$
\left[\left[a,\left[x r_{1}, x r_{2}\right]\right],\left[b,\left[x r_{3}, x r_{4}\right]\right]\right]+\left[\left[b,\left[x r_{1}, x r_{2}\right]\right],\left[a,\left[x r_{3}, x r_{4}\right]\right]\right]=0 .
$$

Denote

$$
\begin{aligned}
F_{1}= & \left(r_{1} x r_{2}-r_{2} x r_{1}\right) b\left[x r_{3}, x r_{4}\right]-\left(r_{1} x r_{2}-r_{2} x r_{1}\right)\left[x r_{3}, x r_{4}\right] b \\
& -\left(r_{3} x r_{4}-r_{4} x r_{3}\right) b\left[x r_{1}, x r_{2}\right]+\left(r_{3} x r_{4}-r_{4} x r_{3}\right)\left[x r_{1}, x r_{2}\right] b, \\
F_{2}= & -\left(r_{3} x r_{4}-r_{4} x r_{3}\right) a\left[x r_{1}, x r_{2}\right]+\left(r_{3} x r_{4}-r_{4} x r_{3}\right)\left[x r_{1}, x r_{2}\right] a \\
& +\left(r_{1} x r_{2}-r_{2} x r_{1}\right) a\left[x r_{3}, x r_{4}\right]-\left(r_{1} x r_{2}-r_{2} x r_{1}\right)\left[x r_{3}, x r_{4}\right] a, \\
F_{3}= & \left(r_{3} x r_{4}-r_{4} x r_{3}\right) b a\left[x r_{1}, x r_{2}\right]-\left(r_{1} x r_{2}-r_{2} x r_{1}\right) b a\left[x r_{3}, x r_{4}\right] \\
+ & \left(r_{1} x r_{2}-r_{2} x r_{1}\right) a\left[x r_{3}, x r_{4}\right] b-\left(r_{3} x r_{4}-r_{4} x r_{3}\right) b\left[x r_{1}, x r_{2}\right] a \\
- & \left(r_{1} x r_{2}-r_{2} x r_{1}\right) b a\left[x r_{3}, x r_{4}\right]+\left(r_{1} x r_{2}-r_{2} x r_{1}\right) b\left[x r_{3}, x r_{4}\right] a \\
+ & \left(r_{3} x r_{4}-r_{4} x r_{3}\right) a b\left[x r_{1}, x r_{2}\right]-\left(r_{3} x r_{4}-r_{4} x r_{3}\right) a\left[x r_{1}, x r_{2}\right] b .
\end{aligned}
$$


Hence (4) is $a x F_{1}+b x F_{2}+x F_{3}=0$. If $\{a x, b x, x\}$ are linearly $C$-independent, then (4) is a nontrivial generalized polynomial identity for $R$, since $F_{1} \neq 0$ in $T$, using $b \notin C$. On the other hand, if there exist $\alpha_{1}, \alpha_{2} \in C$ such that $b x=\alpha_{1} x+\alpha_{2} a x$, it follows that $R$ satisfies

$$
a x F_{1}+\alpha_{1} x F_{2}+\alpha_{2} a x F_{2}+x F_{3}=0
$$

that is, again a nontrivial GPI, because $\{x, a x\}$ are linearly $C$-independent, by the choice of $x$ and since $F_{1}+F_{2} \neq 0$ in $T$, using $a, b \notin C$.

The same argument shows that if $d(I) I \neq 0$, then there exists $x \in I$ such that $\{x, b x\}$ are linearly $C$-independent and $R$ satisfies in any case a nontrivial GPI.

At this point, we need a result that will be useful in the continuation of the note.

Remark 10. Let $R=M_{n}(F)$ be the ring of $n \times n$ matrices over the field $F$, denote by $e_{i j}$ the usual matrix unit with 1 in the $(i, j)$-entry and zero elsewhere. Since there exists a set of matrix units that contains the idempotent generator of a given minimal right ideal, we observe that any minimal right ideal is part of a direct sum of minimal right ideals adding to $R$. In light of this and applying [6, Proposition 5, page 52], we may assume that any minimal right ideal of $R$ is a direct sum of minimal right ideals, each of the form $e_{i i} R$.

LEMmA 11. Let $R=M_{n}(F)$ be the ring of $n \times n$ matrices over the field $F$ of characteristic different from 2 and $n \geq 2$. Let $d$ be a nonzero inner derivation of $R$, and $I$ a nonzero right ideal of $R$. If $a$ is a nonzero element of I such that $\left(d\left(\left[x_{1}, x_{2}\right]\right)\left[x_{3}, x_{4}\right]-d\left(\left[x_{3}, x_{4}\right]\right)\left[x_{1}, x_{2}\right]\right) a=$ 0 , for all $x_{1}, x_{2}, x_{3}, x_{4} \in I$, then either $s_{4}(I, I, I, I) I=0$ or $d$ is induced by an element $b \in R$ such that $(b-\beta) I=0$, for a suitable $\beta \in Z(R)$.

Proof. Let $b$ be an element of $R$ which induces the derivation $d$, that is, $d(x)=[b, x]$, for all $x \in R$. As above, let $e_{i j}$ be the usual matrix unit with 1 in the $(i, j)$-entry and zero elsewhere and write $a=\sum a_{i j} e_{i j}, b=\sum b_{i j} e_{i j}$, with $a_{i j}$ and $b_{i j}$ elements of $F$.

We know that $I$ has a number of uniquely determinated simple components: they are minimal right ideals of $R$ and $I$ is their direct sum. In light of Remark 10, we may write $I=e R$ for some $e=\sum_{i=1}^{t} e_{i i}$ and $t \in\{1,2, \ldots, n\}$. Since $s_{4}(I, I, I, I) I=0$ in case $t \leq 2$, we may suppose that $t \geq 3$.

First of all, we want to prove that $b_{r s}=0$ for all $s \leq t$ and $r \neq s$. To do this, suppose by contradiction that there exist $i \neq j$ such that $b_{i j} \neq 0(j \leq t)$. Without loss of generality, we replace $b$ by $b_{i j}^{-1}\left(b-b_{j j} I_{n}\right)$, where $I_{n}$ is the identity matrix in $M_{n}(F)$ so that we assume $b_{i j}=1$ and $b_{j j}=0$. Moreover $a=e x$ for a suitable $x \in R$.

Let now $k \leq t, k \neq i, j,\left[x_{1}, x_{2}\right]=e_{k i},\left[x_{3}, x_{4}\right]=e_{j i}$. In this case, we have

$$
0=\left(\left[b, e_{k i}\right] e_{j i} a-\left[b, e_{j i}\right] e_{k i}\right) a
$$

and left multiplying by $e_{k k}$,

$$
e_{k i} b e_{j i} a=0
$$

that is, since $b_{i j}=1, e_{i i} a=0$. 
On the other hand, if we choose $\left[x_{1}, x_{2}\right]=e_{k i}$ and $\left[x_{3}, x_{4}\right]=e_{j k}$, we have

$$
0=\left(\left[b, e_{k i}\right] e_{j k}-\left[b, e_{j k}\right] e_{k i}\right) a=\left[b, e_{k i}\right] e_{j k} a=-b_{i j} e_{k k} a
$$

Therefore $e_{r r} a=0$ for all $r \neq j$, that is, $a=e_{j j} a$. Finally, consider $\left[x_{1}, x_{2}\right]=e_{k i}$ and $\left[x_{3}, x_{4}\right]=$ $e_{k k}-e_{j j}$. Then

$$
0=\left(\left[b, e_{k i}\right]\left(e_{k k}-e_{j j}\right)-\left[b, e_{k k}-e_{j j}\right] e_{k i}\right) a=e_{k i} b e_{j j} a,
$$

that is, $e_{j j} a=0$. This implies that $e a=0$, so that $a=0$, a contradiction.

This argument says that if $a \neq 0$, then $b_{i j}=0$ for all $i \neq j, j \leq t$.

Suppose that $(b-\beta) I \neq 0$, for $\beta \in F$. In this case, there exist $1 \leq r, s \leq t$, with $r \neq s$, such that $b_{r r} \neq b_{s s}$.

Let $f$ be the $F$-automorphism of $R$ defined by $f(x)=\left(1-e_{r s}\right) x\left(1+e_{r s}\right)$. Thus we have that $f(x) \in I$, for all $x \in I$ and

$$
\left(\left[f(b),\left[x_{1}, x_{2}\right]\right]\left[x_{3}, x_{4}\right]-\left[f(b),\left[x_{3}, x_{4}\right]\right]\left[x_{1}, x_{2}\right]\right) f(a)=0
$$

for all $x_{1}, x_{2}, x_{3}, x_{4} \in I$. If $a \neq 0$, then $f(a) \neq 0$, and as above, the $(r, s)$-entry of $f(b)$ is zero. On the other hand,

$$
f(b)=\left(1-e_{r s}\right) b\left(1+e_{r s}\right)=b+b_{r r} e_{r s}-b_{s s} e_{r s}
$$

that is, $b_{r r}=b_{s s}$, a contradiction. This means that there exists $\beta \in F$ such that $(b-\beta) I=$ 0 . Denote $b-\beta=p$. Since $b$ and $p$ induce the same inner derivation $d$, we have that $\left(\left[p,\left[x_{1}, x_{2}\right]\right]\left[x_{3}, x_{4}\right]-\left[p,\left[x_{3}, x_{4}\right]\right]\left[x_{1}, x_{2}\right]\right) a=0$ with $p I=0$.

LEMMA 12. Let $R$ be a prime ring of characteristic different from $2, d$ a nonzero inner derivation of $R, I$ a nonzero right ideal of $R$. If $a$ is a nonzero element of I such that $\left(d\left(\left[x_{1}, x_{2}\right]\right)\left[x_{3}\right.\right.$, $\left.\left.x_{4}\right]-d\left(\left[x_{3}, x_{4}\right]\right)\left[x_{1}, x_{2}\right]\right) a=0$, for all $x_{1}, x_{2}, x_{3}, x_{4} \in I$, then either $s_{4}(I, I, I, I) I=0$ or $d$ is induced by an element $b \in R$ such that $(b-\beta) I=0$, for a suitable $\beta \in Z(R)$.

Proof. As a reduction of Lemma 9, we have that if $R$ is not a GPI ring, then we are done. Thus consider the only case when $R$ satisfies a nontrivial generalized polynomial identity.

Thus the Martindale quotient ring $Q$ of $R$ is a primitive ring with nonzero socle $H=$ $\operatorname{Soc}(Q) . H$ is a simple ring with minimal right ideals. Let $D$ be the associated division ring of $H$, by [11] $D$ is a simple central algebra finite-dimensional over $C=Z(Q)$. Thus $H \otimes_{C} F$ is a simple ring with minimal right ideals, with $F$ an algebraic closure of $C$. Let $b$ be an element of $R$ which induces the derivation $d$. Moreover $\left(\left[b,\left[x_{1}, x_{2}\right]\right]\left[x_{3}, x_{4}\right]-\right.$ $\left.\left[b,\left[x_{3}, x_{4}\right]\right]\left[x_{1}, x_{2}\right]\right) a=0$, for all $x_{1}, x_{2}, x_{3}, x_{4} \in I H \otimes_{C} F$ (see, e.g., [4, Theorem 2]). Notice that if $C$ is finite, we choose $F=C$.

Now we claim that for any $c \in I H$, there exists $\beta \in C$ with $(b-\beta) c=0$. If not, then for some $c \in I H,(b-\beta) c \neq 0$ for all $\beta \in C$, so in particular $b c \neq 0$. Since $H$ is regular [5], there exists $g^{2}=g \in I H$, such that $c \in g I H$, and $e^{2}=e \in H \otimes_{C} F$, such that

$$
g, b g, g b, a, c, b c, c b \in e\left(H \otimes_{C} F\right) e \cong M_{n}(F), \quad n \geq 3 .
$$


Let $x_{1}, x_{2}, x_{3}, x_{4} \in g e\left(H \otimes_{C} F\right) e$ and $a=e a e \neq 0$, then

$$
0=e\left(\left[b,\left[x_{1}, x_{2}\right]\right]\left[x_{3}, x_{4}\right]-\left[b,\left[x_{3}, x_{4}\right]\right]\left[x_{1}, x_{2}\right]\right) e a e .
$$

Applying Lemma 11, we have that $e(b-\lambda) e c=0$ for $\lambda \in C$, so $(b-\beta) c=0$, contradicting the choice of $c$.

As in the proof of Lemma 9, by [10, Lemma 3], we conclude that there exists $\beta \in C$ such that $(b-\beta) I=0$.

LEMma 13. If $\delta$ and $d$ are both inner derivations, then the theorem holds.

Proof. By Remark 2, we assume that $[\delta(u), d(v)]+[d(u), \delta(v)]=0$, for any $u, v \in[I, I]$. Let $a, b \in Q$ such that $\delta(x)=[a, x]$ and $d(x)=[b, x]$, for all $x \in R$. Since in light of Lemma $9, R$ satisfies a nontrivial GPI, then without loss of generality, $R$ is simple and equal to its own socle and $I R=I$. In fact, $Q$ has nonzero socle $H$ with nonzero right ideal $J=I H[11]$. Note that $H$ is simple, $J=J H$, and $J$ satisfies the same basic conditions as $I$. Now just replace $R$ by $H, I$ by $J$, and we are done.

Recall that $s_{4}\left(c_{1}, c_{2}, c_{3}, c_{4}\right) c_{5} \neq 0$ and either $\delta\left(c_{6}\right) c_{7} \neq 0$ or $d\left(c_{8}\right) c_{9} \neq 0$. By the regularity of $R$, there exists an element $e^{2}=e \in I R$ such that $e R=c_{1} R+c_{2} R+c_{3} R+c_{4} R+c_{5} R+$ $c_{6} R+c_{7} R+c_{8} R+c_{9} R$ and $e c_{i}=c_{i}$, for $i=1, \ldots, 9$. We note that $s_{4}(e R e, e R e, e R e, e R e) \neq 0$ (and $\operatorname{dim}_{C}(e R e) \geq 9$ ).

Let $x, y, z \in R$, so

$$
[[a,[e, e x(1-e)]],[b,[e y, e z]]]+[[b,[e, e x(1-e)]],[a,[e y, e z]]]=0 .
$$

Denote $A=(1-e) a e, B=(1-e) b e$. Assume that $A=0$ but $B \neq 0$. Consider first the case when $\{1-e,(1-e) a\}$ are linearly $C$-independent. Equation (15), multiplied on the left by $(1-e)$, says that

$$
-(1-e) b[e y, e z] a e x(1-e)+(1-e) b[e y, e z] \operatorname{ex}(1-e) a=0 .
$$

By Remark 8 and since $\{1-e,(1-e) a\}$ are linearly $C$-independent, it follows that there exists $\lambda_{1} \in C$ such that $-(1-e) b[e y, e z] a e=\lambda_{1}(1-e) b[e y, e z] e$.

Therefore

$$
(1-e) b[e y, e z] \operatorname{ex\lambda } \lambda_{1}(1-e)+(1-e) b[e y, e z] \operatorname{ex}(1-e) a=0,
$$

which implies that $(1-e) b[e y, e z] e=0$, again since $\{1-e,(1-e) a\}$ are linearly $C$-independent. If we denote $T=e R,(1-e) b[T, T] T=0$ forces $(1-e) b T[T, T] T=0$, so either $(1-e) b T=0$ or $[T, T] T=0$. Thus we have that either $B=(1-e) b e=0$ or $\left[x_{1}, x_{2}\right] x_{3}$ is an identity for $e R$. In this last case, a fortiori $s_{4}\left(x_{1}, x_{2}, x_{3}, x_{4}\right) x_{5}$ is an identity for $e R$. In both cases, we have a contradiction, since we suppose that $B \neq 0$ and $s_{4}\left(c_{1}, c_{2}, c_{3}, c_{4}\right) c_{5} \neq 0$.

Suppose now that $(1-e) a=\lambda(1-e)$, for some $\lambda \in C$. Equation (16) says that

$$
-(1-e) b[e y, e z] a e x(1-e)+\lambda(1-e) b[e y, e z] \operatorname{ex}(1-e)=0
$$


and so

$$
-(1-e) b[e y, e z] a e+\lambda(1-e) b[e y, e z] e=0
$$

that is, for $a^{\prime}=\lambda e-a e$,

$$
(1-e) b[e y, e z] a^{\prime}=0 .
$$

Denote $U=[e y, e z] a^{\prime}$. Since $(1-e) b e\left[U x_{1}, e x_{2}\right] a^{\prime}=0$, for all $x_{1}, x_{2} \in R$, it follows that $(1-e) b e x_{2} U x_{1} a^{\prime}=0$, and so either $a^{\prime}=0$ or $U=0$. Again denote $T=e R$. If $U=0$, we have $[T, T] a^{\prime}=0$, so that $[T, T] T a^{\prime}=0$, which implies either $a^{\prime}=0$ or $[T, T] T=0$. Since $[e R, e R] e \neq 0$, we have $a e=\lambda e$ in any case.

All the previous arguments say that $(a-\lambda) e=0$. Replacing $a$ by $a-\lambda=a^{\prime \prime}$, since they induce the same inner derivation, we may assume that for all $x, y, z, t \in R$,

$$
\left[\left[a^{\prime \prime},[e x, e y]\right],[b,[e z, e t]]\right]+\left[[b,[e x, e y]],\left[a^{\prime \prime},[e z, e t]\right]\right]=0 .
$$

Left multiplying $(21)$ by $(1-e)$, we have

$$
(1-e) b e[[e z, e t],[e x, e y]] a^{\prime \prime}=0,
$$

in particular

$$
0=(1-e) b e[[e z, e t],[\operatorname{ex}, e y(1-e)]] a^{\prime \prime}=(1-e) b e[e z, e t] \operatorname{exey}(1-e) a^{\prime \prime},
$$

and by the previous same argument, $(1-e) a^{\prime \prime}=0$, that is, $a^{\prime \prime}=e a^{\prime \prime}$. In light of this, by (22),

$$
(e R(1-e) b e)[[\text { eze,ete }],[\text { exe, eye }]]\left(e a^{\prime \prime} R e\right)=0 .
$$

Let $G$ be the subgroup of $e$ Re generated by the polynomial [[eze,ete], [exe,eye]]. It is easy to see that $G$ is a noncentral Lie ideal of $e$ Re. In this condition, it is well known that $[e R e, e R e] \subseteq G$, and so $e R(1-e) b e[e R e, e R e] e a^{\prime \prime} R e=0$.

Consider now the simple Artinian ring $e$ Re, then we have that

$$
e R(1-e) b e\left[e x_{1} e, e x_{2} e\right]\left(e a^{\prime \prime} R e\right)=0 \quad \forall x_{1}, x_{2} \in R .
$$

Let $U=\left[e x_{1} e, e x_{2} e\right]\left(e a^{\prime \prime} R e\right)$, so $e R(1-e) b e U=0$. Since

$$
(e R(1-e) b e)\left[U e x_{1} e, e x_{2} e\right]\left(e a^{\prime \prime} R e\right)=0,
$$

then

$$
(e R(1-e) b e) x_{2} U e x_{1}\left(e a^{\prime \prime} R e\right)=0 .
$$

It follows that if $(1-e) b e \neq 0$, then $a^{\prime \prime}=0$, that is, $a=\lambda \in C$, a contradiction. Thus the conclusion is that if $A=(1-e) a e=0$, then $B=(1-e) b e=0$.

Similarly, $A=(1-e) a e=0$ follows from $B=(1-e) b e=0$. 
Now we assume that neither $A=0$ nor $B=0$, and proceed to get contradictions, proving that $A=B=0$.

Left multiplying $(15)$ by $(1-e)$ and right multiplying by $e$, we get

$$
\begin{aligned}
& (1-e) \operatorname{aex}(1-e) b[e y, e z] e+(1-e) b[e y, e z] \operatorname{ex}(1-e) a e \\
& +(1-e) b \operatorname{ex}(1-e) a[e y, e z] e+(1-e) a[e y, e z] \operatorname{ex}(1-e) b e=0 .
\end{aligned}
$$

If we denote $A^{\prime}=A[y e, z e], B^{\prime}=B[y e, z e]$, it follows that

$$
A x B^{\prime}+B^{\prime} x A+B x A^{\prime}+A^{\prime} x B=0
$$

Consider now the case when $A, B$ are linearly $C$-independent.

In light of Remark 8 and (29), it follows that there exist $\alpha_{1}, \alpha_{2}, \alpha_{3}, \alpha_{4}$ in $C$ such that $B^{\prime}=\alpha_{1} A+\alpha_{2} B, A^{\prime}=\alpha_{3} A+\alpha_{4} B$. So we rewrite (29) as follows:

$$
2 \alpha_{1} A x A+2 \alpha_{4} B x B+\left(\alpha_{2}+\alpha_{3}\right) A x B+\left(\alpha_{2}+\alpha_{3}\right) B x A=0
$$

that is,

$$
A x\left(2 \alpha_{1} A+\left(\alpha_{2}+\alpha_{3}\right) B\right)+B x\left(2 \alpha_{4} B+\left(\alpha_{2}+\alpha_{3}\right) A\right)=0 .
$$

Since $A, B$ are $C$-independent, by (31) and again Remark 8, it follows that $2 \alpha_{1} A+\left(\alpha_{2}+\right.$ $\left.\alpha_{3}\right) B=0$ and $2 \alpha_{4} B+\left(\alpha_{2}+\alpha_{3}\right) A=0$, so the independence of $A$ and $B$ forces $\alpha_{1}=\alpha_{4}=$ $\alpha_{2}+\alpha_{3}=0$.

Therefore we have that $B[e R e, e R e] \subseteq C B$. Notice that $B[e R e, e R e] \neq 0$. In fact, if $B[e R e, e R e]=0$, since $[e R e, e R e] \neq(0)$ is a noncentral Lie ideal of the simple Artinian ring $e R e$, the contradiction $B=0$ is immediate.

Let $u, v \in[e R e, e R e]$. Hence there exist $\omega_{1}, \omega_{2}, 0 \neq \omega \in C$ such that

$$
B[u, v]=\omega B \neq 0, \quad B u=\omega_{1} B, \quad B v=\omega_{2} B
$$

and by calculation we get the contradiction

$$
0 \neq \omega B=B[u, v]=0
$$

Hence we may assume that $A$ and $B$ are linearly $C$-dependent, say $A=\alpha B$, for $0 \neq \alpha \in C$, so also $A^{\prime}=\alpha B^{\prime}$. Equation (29) is now $2 \alpha B x B^{\prime}+2 \alpha B^{\prime} x B=0$, and it follows that $B$ and $B^{\prime}$ must be linearly $C$-dependent, so that $B x B=0$ and $B=B^{\prime}=0$.

Therefore in any case, we have that if $s_{4}(e R, e R, e R, e R) e \neq 0$, then $(1-e) b e=(1-$ $e) a e=0$.

Let $J=e R, \bar{J}=J / J \cap l_{R}(J) ; \bar{J}$ is a prime $C$-algebra. Since $d(J) \subseteq J$ and $\delta(J) \subseteq J, d$ and $\delta$ induce on $\bar{J}$ the following two derivations:

$$
\begin{array}{ll}
\bar{d}: \bar{J} \longrightarrow \bar{J} \quad \text { such that } \bar{d}(\bar{x})=\overline{d(x)} \\
\bar{\delta}: \bar{J} \longrightarrow \bar{J} \quad \text { such that } \bar{\delta}(\bar{x})=\overline{\delta(x)}
\end{array}
$$


Therefore, we have

$$
0=\left[\overline{\delta\left(\left[r_{1}, r_{2}\right]\right)}, \overline{d\left[r_{3}, r_{4}\right]}\right]+\left[\overline{d\left(\left[r_{1}, r_{2}\right]\right)}, \overline{\delta\left[r_{3}, r_{4}\right]}\right]
$$

for all $\overline{r_{1}}, \overline{r_{2}}, \overline{r_{3}}, \overline{r_{4}} \in \bar{J}$. By Lemma 4 , we have that one of the following holds:

$$
\bar{\delta}=\overline{0}, \quad \bar{d}=\overline{0}, \quad \bar{J} \text { is commutative. }
$$

Since $s_{4}(J, J, J, J) J \neq 0$, the last case cannot occur. On the other hand, now we prove that also the other cases lead us to contradictions.

Suppose that the first case occurs, that is, $\delta(J) J=0$. By the lemma in [3], there exists an element $q=a-\alpha \in Q$, with $\alpha \in C$, such that $(a-\alpha) J=0$. Moreover $a$ and $q$ induce the same inner derivation $\delta$, so that we have

$$
\left(\left[b,\left[x_{1}, x_{2}\right]\right]\left[x_{3}, x_{4}\right]-\left[b,\left[x_{3}, x_{4}\right]\right]\left[x_{1}, x_{2}\right]\right) q=0 \quad \forall x_{1}, x_{2}, x_{3}, x_{4} \in J
$$

In particular, for any $r \in R$, choose $\left[x_{1}, x_{2}\right]=[e, \operatorname{er}(1-e)]=\operatorname{er}(1-e)$. From (37), it follows that

$$
\left[b,\left[x_{3}, x_{4}\right]\right] e R(1-e) q=0
$$

If $(1-e) q=0$, we have $q=e q \in J$. Under this condition, by Lemma 12 , it follows from (37) that either $q=0$, which implies the contradiction $a \in C$ and $\delta=0$, or $(b-\beta) J=0$ for a suitable $\beta \in C$, that is, $d(e R) e R=0$. So consider the case when $\left[b,\left[x_{3}, x_{4}\right]\right] e=0$ for all $x_{3}, x_{4} \in J$, and remember that $b e=e b e$. This implies that $\left[e b e,\left[y_{1}, y_{2}\right]\right]=0$ for all $y_{1}, y_{2} \in$ $e R e$, that is, either $e R e$ is a commutative central simple algebra or $e b e \in C e$. In the first case, we have the contradiction $0=s_{4}\left(e c_{1}, e c_{2}, e c_{3}, e c_{4}\right) e c_{5}=s_{4}\left(c_{1}, c_{2}, c_{3}, c_{4}\right) c_{5} \neq 0$. In the second one, we get again $d(e R) e R=0$. Therefore we conclude that in any case, $\delta(e R) e R=$ $d(e R) e R=0$, which is again a contradiction because of $\delta\left(c_{6}\right) c_{7} \neq 0$ or $d\left(c_{8}\right) c_{9} \neq 0$.

Obviously by a similar argument and (36), we are also finished when $d(J) J=0$.

For the proof of the main theorem, we need the following results.

LEMma 14. Let $R$ be a prime ring of characteristic different from 2 and $I$ a nonzero right ideal of $R$. If for any $x_{1}, x_{2}, x_{3}, x_{4} \in I,\left[\left[x_{1}, x_{2}\right],\left[x_{3}, x_{4}\right]\right]=0$, then $[I, I] I=0$.

Proof. First note that if $[y,[I, I]]=0$, for some $y \in R$, then, for any $s, t \in I$, we have $0=$ $[y,[s t, t]]=[s, t][y, t]$. In particular, for any $x \in I R, 0=[s x, t][y, t]=[s, t] x[y, t]$, that is $[s, t] I R[y, t]=0$. By the primeness of $R$, we have that either $[s, t] I=0$, that is, $[I, I] I=0$, or $[y, I]=0$. In this last case, $0=[y, I R]=I[y, R]$ forcing $y \in Z(R)$.

Therefore, if we assume that $[I, I] I \neq 0$, the assumption $[[I, I],[I, I]]=0$ forces $0 \neq$ $[I, I] \subseteq Z(R)$. Let $s, t \in I$ be such that $[s, t] I \neq 0$ and $[s, t] \in Z(R)$. Then $2 s[s, t]=\left[s^{2}, t\right] \in$ $Z(R)$, so $[s, t] \neq 0$ forces $s \in Z(R)$ and we have the contradiction $[s, I]=0$.

LEMma 15. Let $R$ be a noncommutative prime ring of characteristic different from 2, $q$ a noncentral element of $R$, and $I$ a nonzero right ideal of $R$. If for any $x_{1}, x_{2}, x_{3}, x_{4} \in I$, $\left[\left[q,\left[x_{1}, x_{2}\right]\right],\left[x_{3}, x_{4}\right]\right]=0$, then $[I, I] I=0$. 
Proof. Suppose that $[I, I] I \neq 0$. As in Lemma 14, first we recall that the condition $[y,[I$, $I]]=0$ forces $y \in Z(R)$. This means that $[q,[I, I]] \subseteq Z(R)$, since $[[q,[I, I]],[I, I]]=0$. Moreover we may assume that $[q,[I, I]] \neq 0$, if not, then we are finished by Lemma 14 .

Note that from $[q,[I, I]] \subseteq Z(R)$, it follows that $[q,[[I, I], I]] \subseteq Z(R)$. Expanding this yields $[[I, I],[q, I]] \subseteq Z(R)$. Since for all $x \in I$, we have $[[I, I],[q, x q]] \subseteq Z(R)$, then $[[I, I]$, $[q, I] q] \subseteq Z(R)$. Hence

$$
0=[[[I, I],[q, I] q], q]=[q,[q, I]][q,[I, I]]
$$

Since the second factor is nonzero and central, we have $[q,[q, I]]=0$, which implies that for all $x, y \in I$,

$$
0=[q,[q, x y]]=[q,[q, x] y+x[q, y]]=2[q, x][q, y] .
$$

This means that $[q, I][q, I]=0$ and a fortiori $[q,[I, I]][q,[I, I]]=0$ giving the contradiction $[q,[I, I]]=0$.

We are ready to prove the following main result.

THeOREM 16. Let $R$ be a prime ring of characteristic different from 2, I a nonzero right ideal of $R, d$ and $\delta$ nonzero derivations of $R, s_{4}\left(x_{1}, \ldots, x_{4}\right)$ the standard identity of degree 4. If the composition $(d \delta)$ is a Lie derivation of $[I, I]$ into $R$, then either $s_{4}(I, I, I, I) I=0$ or $\delta(I) I=d(I) I=0$.

Proof. By Remark 2, we assume that $[\delta(u), d(v)]+[d(u), \delta(v)]=0$, for any $u, v \in[I, I]$. Suppose by contradiction that there exist $c_{1}, c_{2}, c_{3}, c_{4}, c_{5}, c_{6}, c_{7}, c_{8}, c_{9}$ in $I$ such that $s_{4}\left(c_{1}, c_{2}, c_{3}, c_{4}\right) c_{5} \neq 0$ and either $\delta\left(c_{6}\right) c_{7} \neq 0$ or $d\left(c_{8}\right) c_{9} \neq 0$.

First suppose that $\delta$ and $d$ are $C$-independent modulo $D_{\text {int }}$.

Let $t_{1}, t_{2}, t_{3}, t_{4} \in I$, by assumption, $R$ satisfies

$$
\begin{gathered}
{\left[\left[\delta\left(t_{1} x_{1}\right), t_{2} x_{2}\right]+\left[t_{1} x_{1}, \delta\left(t_{2} x_{2}\right)\right],\left[d\left(t_{3} x_{3}\right), t_{4} x_{4}\right]+\left[t_{3} x_{3}, d\left(t_{4} x_{4}\right)\right]\right]} \\
+\left[\left[d\left(t_{1} x_{1}\right), t_{2} x_{2}\right]+\left[t_{1} x_{1}, d\left(t_{2} x_{2}\right)\right],\left[\delta\left(t_{3} x_{3}\right), t_{4} x_{4}\right]+\left[t_{3} x_{3}, \delta\left(t_{4} x_{4}\right)\right]\right] \\
=\left[\left[\delta\left(t_{1}\right) x_{1}+t_{1} \delta\left(x_{1}\right), t_{2} x_{2}\right]+\left[t_{1} x_{1}, \delta\left(t_{2}\right) x_{2}+t_{2} \delta\left(x_{2}\right)\right],\right. \\
\left.\left[d\left(t_{3}\right) x_{3}+t_{3} d\left(x_{3}\right), t_{4} x_{4}\right]+\left[t_{3} x_{3}, d\left(t_{4}\right) x_{4}+t_{4} d\left(x_{4}\right)\right]\right] \\
+\left[\left[d\left(t_{1}\right) x_{1}+t_{1} d\left(x_{1}\right), t_{2} x_{2}\right]+\left[t_{1} x_{1}, d\left(t_{2}\right) x_{2}+t_{2} d\left(x_{2}\right)\right],\right. \\
\left.\quad\left[\delta\left(t_{3}\right) x_{3}+t_{3} \delta\left(x_{3}\right), t_{4} x_{4}\right]+\left[t_{3} x_{3}, \delta\left(t_{4}\right) x_{4}+t_{4} \delta\left(x_{4}\right)\right]\right]=0 .
\end{gathered}
$$

By Kharchenko's theorem [7], $R$ satisfies the generalized polynomial identity

$$
\begin{aligned}
& {\left[\left[\delta\left(t_{1}\right) x_{1}+t_{1} y_{1}, t_{2} x_{2}\right]+\left[t_{1} x_{1}, \delta\left(t_{2}\right) x_{2}+t_{2} y_{2}\right],\left[d\left(t_{3}\right) x_{3}+t_{3} z_{3}, t_{4} x_{4}\right]+\left[t_{3} x_{3}, d\left(t_{4}\right) x_{4}+t_{4} z_{4}\right]\right]} \\
& +\left[\left[d\left(t_{1}\right) x_{1}+t_{1} z_{1}, t_{2} x_{2}\right]+\left[t_{1} x_{1}, d\left(t_{2}\right) x_{2}+t_{2} z_{2}\right],\left[\delta\left(t_{3}\right) x_{3}+t_{3} y_{3}, t_{4} x_{4}\right]+\left[t_{3} x_{3}, \delta\left(t_{4}\right) x_{4}+t_{4} y_{4}\right]\right]
\end{aligned}
$$


in particular $R$ satisfies $\left[\left[t_{1} y_{1}, t_{2} x_{2}\right],\left[t_{3} x_{3}, t_{4} z_{4}\right]\right]$, so $Q$ satisfies this as well, and for all $y_{1}=$ $x_{2}=x_{3}=z_{4}=1 \in Q$, it follows that $[[I, I],[I, I]]=0$. Thus by Lemma 14 , we conclude that $[I, I] I=0$, that is, $s_{4}(I, I, I, I) I=0$, which contradicts $s_{4}\left(c_{1}, c_{2}, c_{3}, c_{4}\right) c_{5} \neq 0$.

Now let $\delta$ and $d$ be $C$-dependent modulo $D_{\text {int }}$. There exist $\gamma_{1}, \gamma_{2} \in C$, such that $\gamma_{1} \delta+$ $\gamma_{2} d \in D_{\text {int }}$, and by Lemma 13 , it is clear that at most one of the two derivations can be inner.

Without loss of generality, we may assume that $\gamma_{1} \neq 0$, so that $\delta=\alpha d+a d(q)$, for $\alpha \in C$ and $a d(q)$ the inner derivation induced by the element $q \in Q$.

If $d$ is inner, then also $\delta$ is inner, and we have that $d$ is an outer derivation of $R$. Let $t_{1}, t_{2}, t_{3}, t_{4} \in I, R$ satisfies

$$
\begin{aligned}
\alpha\left[\left[d\left(t_{1} x_{1}\right), t_{2} x_{2}\right]+\left[t_{1} x_{1}, d\left(t_{2} x_{2}\right)\right],\left[d\left(t_{3} x_{3}\right), t_{4} x_{4}\right]+\left[t_{3} x_{3}, d\left(t_{4} x_{4}\right)\right]\right] & +\left[\left[q,\left[t_{1} x_{1}, t_{2} x_{2}\right]\right],\left[d\left(t_{3} x_{3}\right), t_{4} x_{4}\right]+\left[t_{3} x_{3}, d\left(t_{4} x_{4}\right)\right]\right] \\
+ & \alpha\left[\left[d\left(t_{1} x_{1}\right), t_{2} x_{2}\right]+\left[t_{1} x_{1}, d\left(t_{2} x_{2}\right)\right],\left[d\left(t_{3} x_{3}\right), t_{4} x_{4}\right]+\left[t_{3} x_{3}, d\left(t_{4} x_{4}\right)\right]\right] \\
+ & {\left[\left[d\left(t_{1} x_{1}\right), t_{2} x_{2}\right]+\left[t_{1} x_{1}, d\left(t_{2} x_{2}\right)\right],\left[q,\left[t_{3} x_{3}, t_{4} x_{4}\right]\right]\right] } \\
=\alpha[ & \left.d\left(t_{1}\right) x_{1}+t_{1} d\left(x_{1}\right), t_{2} x_{2}\right]+\left[t_{1} x_{1}, d\left(t_{2}\right) x_{2}+t_{2} d\left(x_{2}\right)\right], \\
& \left.\quad\left[d\left(t_{3}\right) x_{3}+t_{3} d\left(x_{3}\right), t_{4} x_{4}\right]+\left[t_{3} x_{3}, d\left(t_{4}\right) x_{4}+t_{4} d\left(x_{4}\right)\right]\right] \\
+ & {\left[\left[q,\left[t_{1} x_{1}, t_{2} x_{2}\right]\right],\left[d\left(t_{3}\right) x_{3}+t_{3} d\left(x_{3}\right), t_{4} x_{4}\right]+\left[t_{3} x_{3}, d\left(t_{4}\right) x_{4}+t_{4} d\left(x_{4}\right)\right]\right] } \\
+ & \alpha\left[\left[d\left(t_{1}\right) x_{1}+t_{1} d\left(x_{1}\right), t_{2} x_{2}\right]+\left[t_{1} x_{1}, d\left(t_{2}\right) x_{2}+t_{2} d\left(x_{2}\right)\right],\right. \\
& \left.\quad\left[d\left(t_{3}\right) x_{3}+t_{3} d\left(x_{3}\right), t_{4} x_{4}\right]+\left[t_{3} x_{3}, d\left(t_{4}\right) x_{4}+t_{4} d\left(x_{4}\right)\right]\right] \\
+ & {\left[\left[d\left(t_{1}\right) x_{1}+t_{1} d\left(x_{1}\right), t_{2} x_{2}\right]+\left[t_{1} x_{1}, d\left(t_{2}\right) x_{2}+t_{2} d\left(x_{2}\right)\right],\left[q,\left[t_{3} x_{3}, t_{4} x_{4}\right]\right]\right], }
\end{aligned}
$$

and so the Kharchenko theorem shows that $R$ satisfies

$$
\begin{aligned}
& \alpha\left[\left[d\left(t_{1}\right) x_{1}+t_{1} y_{1}, t_{2} x_{2}\right]+\left[t_{1} x_{1}, d\left(t_{2}\right) x_{2}+t_{2} y_{2}\right],\left[d\left(t_{3}\right) x_{3}+t_{3} y_{3}, t_{4} x_{4}\right]+\left[t_{3} x_{3}, d\left(t_{4}\right) x_{4}+t_{4} y_{4}\right]\right] \\
& +\left[\left[q,\left[t_{1} x_{1}, t_{2} x_{2}\right]\right],\left[d\left(t_{3}\right) x_{3}+t_{3} y_{3}, t_{4} x_{4}\right]+\left[t_{3} x_{3}, d\left(t_{4}\right) x_{4}+t_{4} y_{4}\right]\right] \\
& +\alpha\left[\left[d\left(t_{1}\right) x_{1}+t_{1} y_{1}, t_{2} x_{2}\right]+\left[t_{1} x_{1}, d\left(t_{2}\right) x_{2}+t_{2} y_{2}\right],\left[d\left(t_{3}\right) x_{3}+t_{3} y_{3}, t_{4} x_{4}\right]+\left[t_{3} x_{3}, d\left(t_{4}\right) x_{4}+t_{4} y_{4}\right]\right] \\
& +\left[\left[d\left(t_{1}\right) x_{1}+t_{1} y_{1}, t_{2} x_{2}\right]+\left[t_{1} x_{1}, d\left(t_{2}\right) x_{2}+t_{2} y_{2}\right],\left[q,\left[t_{3} x_{3}, t_{4} x_{4}\right]\right]\right] .
\end{aligned}
$$

In case $\alpha \neq 0$, for $x_{1}=x_{4}=0$ in (44), we have that $R$ satisfies

$$
2 \alpha\left[\left[t_{1} y_{1}, t_{2} x_{2}\right],\left[t_{3} x_{3}, t_{4} y_{4}\right]\right]
$$

so $Q$ satisfies this as well and for all $y_{1}=x_{2}=x_{3}=y_{4}=1 \in Q$, it follows that $2 \alpha[[I, I]$, $[I, I]]=0$. Hence, if $\alpha \neq 0$, by Lemma 14, we have the contradiction $[I, I] I=0$. 
Now let $\alpha=0$. In this case for $x_{4}=0$ in (44), we have that $R$ satisfies

$$
\left[\left[q,\left[t_{1} x_{1}, t_{2} x_{2}\right]\right],\left[t_{3} x_{3}, t_{4} y_{4}\right]\right] .
$$

As above $Q$ satisifes this and, taking $x_{1}, x_{2}, x_{3}, y_{4}=1$, it follows that

$$
[[q,[I, I]],[I, I]]=0 .
$$

Then, by Lemma 15, we conclude again with the contradiction $[I, I] I=0$.

Similarly, when $\gamma_{2} \neq 0$, then $d=\beta \delta+a d(q)$, for some $\beta \in C$, and mimicking the argument above gives another contradiction.

\section{Acknowledgment}

The author wishes to thank the referee for her/his valuable suggestions on how to improve the paper.

\section{References}

[1] K. I. Beidar, Rings with generalized identities. III, Moscow University Mathematics Bulletin 33 (1978), no. 4, 53-58.

[2] K. I. Beidar, W. S. Martindale III, and A. V. Mikhalev, Rings with Generalized Identities, Monographs and Textbooks in Pure and Applied Mathematics, vol. 196, Marcel Dekker, New York, 1996.

[3] M. Brešar, One-sided ideals and derivations of prime rings, Proceedings of the American Mathematical Society 122 (1994), no. 4, 979-983.

[4] C.-L. Chuang, GPIs having coefficients in Utumi quotient rings, Proceedings of the American Mathematical Society 103 (1988), no. 3, 723-728.

[5] C. Faith and Y. Utumi, On a new proof of Litoff's theorem, Acta Mathematica Academiae Scientiarum Hungaricae 14 (1963), no. 3-4, 369-371.

[6] N. Jacobson, Structure of Rings, American Mathematical Society Colloquium Publications, vol. 37, American Mathematical Society, Rhode Island, 1964.

[7] V. K. Kharchenko, Differential identities of prime rings, Algebra and Logic 17 (1978), no. 2, 155168.

[8] C. Lanski, Differential identities, Lie ideals, and Posner's theorems, Pacific Journal of Mathematics 134 (1988), no. 2, 275-297.

[9] T.-K. Lee, Semiprime rings with differential identities, Bulletin of the Institute of Mathematics. Academia Sinica 20 (1992), no. 1, 27-38.

[10] Left annihilators characterized by GPIs, Transactions of the American Mathematical Society 347 (1995), no. 8, 3159-3165.

[11] W. S. Martindale III, Prime rings satisfying a generalized polynomial identity, Journal of Algebra 12 (1969), no. 4, 576-584.

[12] E. C. Posner, Derivations in prime rings, Proceedings of the American Mathematical Society 8 (1957), no. 6, 1093-1100.

V. De Filippis: Dipartimento di Matematica, Universitá di Messina, Salita Sperone 31,

Messina 98166, Italy

E-mail address: defilippis@unime.it 


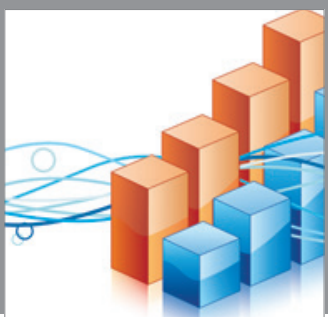

Advances in

Operations Research

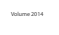

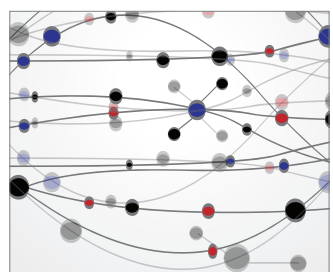

\section{The Scientific} World Journal
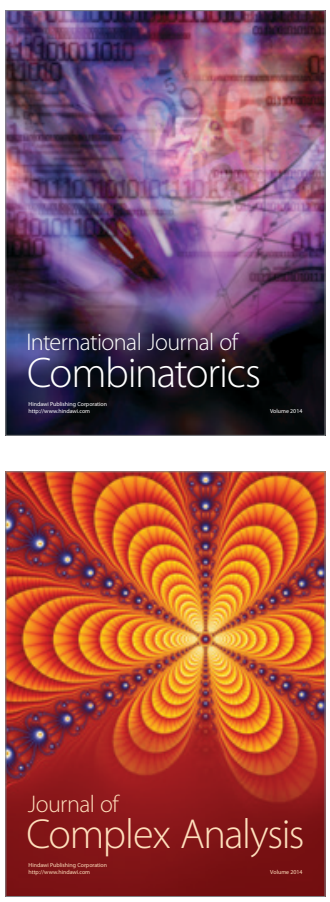

International Journal of

Mathematics and

Mathematical

Sciences
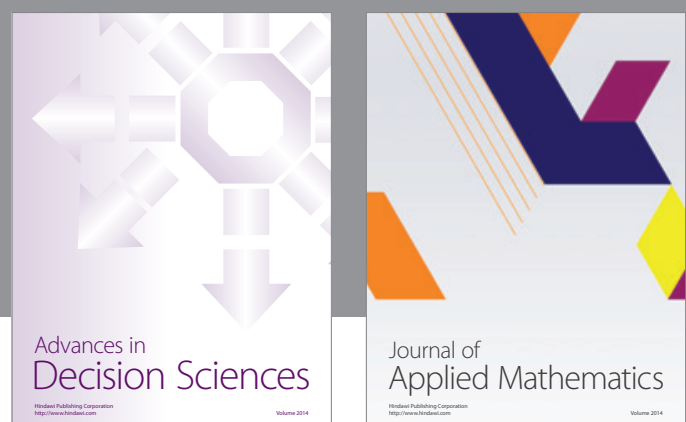

Journal of

Applied Mathematics
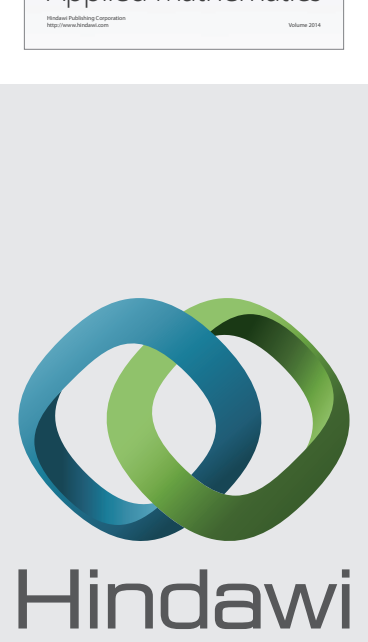

Submit your manuscripts at http://www.hindawi.com
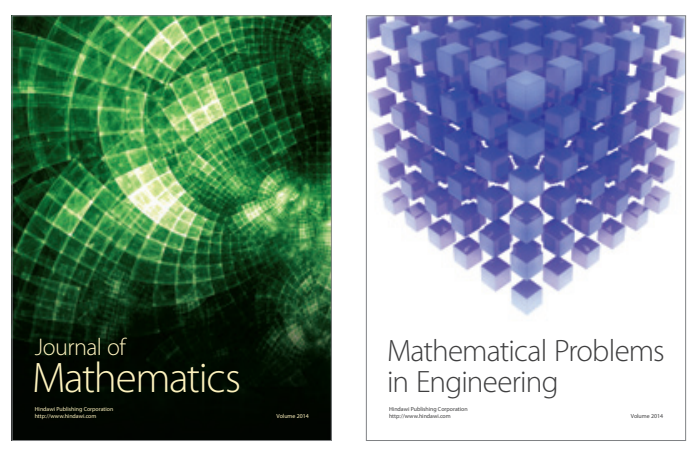

Mathematical Problems in Engineering
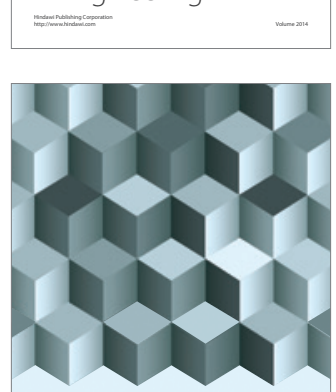

Journal of

Function Spaces
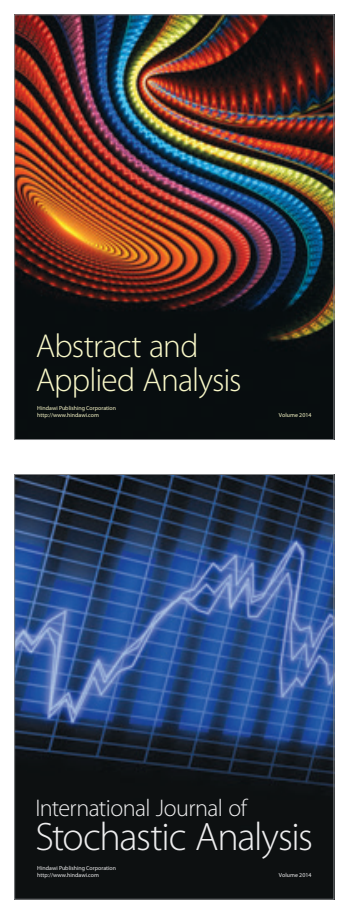

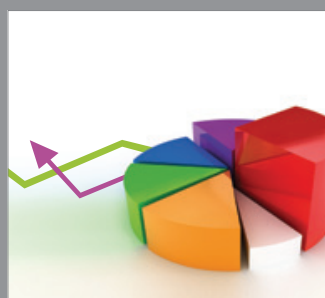

ournal of

Probability and Statistics

Promensencen
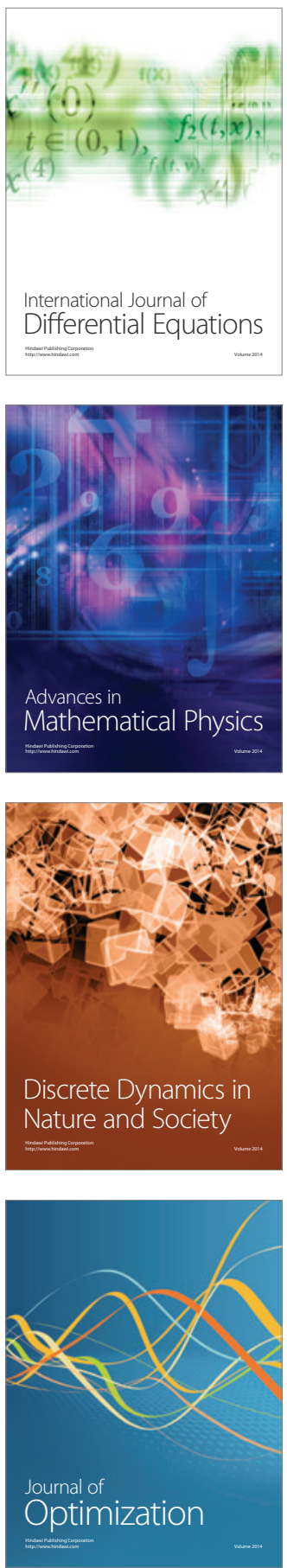